\title{
INCLUSIÓN DE COMPOST DE CAMA PROFUNDA PORCINA EN ALIMENTACIÓN DE VAQUILLAS MESTIZAS DE DOBLE PROPÓSITO
}

\section{INCLUSION OF SWINE DEEP-BED COMPOST IN THE DIET OF DUAL-PURPOSE MONGREL HEIFERS}

\author{
Carlos Octavio Larrea Izurieta, Jorge Ignacio Macías Andrade, Carlos Jesús Alcívar Cedeño, Luis Stiven Marcillo Cevallos \\ Carrera de Medicina Veterinaria, Escuela Superior Politécnica Agropecuaria de Manabí Manuel Félix López, Campus \\ Politécnico El Limón, km 2.7 vía Calceta-El Morro-El Limón, sector el Gramal.
}

Email: colirbba@hotmail.com

\section{Información del artículo}

Tipo de artículo: Artículo original

Recibido:

24/08/2020

Aceptado:

16/12/2020

Licencia:

CC BY-NC-SA 4.0

Revista

ESPAMCIENCIA

11(2):101-107

DOI:

https://doi.org/10.51

260/revista_espamci

encia.v11i2.222

\section{Resumen}

Se evaluó la inclusión de compost de cama profunda (CP) de cascarilla de arroz y porquinaza en la alimentación de vaquillas cruzadas de doble propósito. Para ello se utilizaron 18 vaquillas alimentadas con CP procesada en 12 meses obtenidos de la Unidad de Docencia, Investigación y Vinculación Hato Porcino de la Escuela Superior Politécnica Agropecuaria de Manabí "Manuel Félix López". Se aplicó un DCA con arreglo bifactorial [primer factor: edad (menor a 25 meses de edad y mayor a 25 meses de edad y preñadas de diferente tiempo de gestación); segundo factor: niveles de compost de CP $(0 \%, 10 \%$ y $20 \%$ )], se consideró como covariable al peso inicial. La composición nutricional del compost de CP fue determinada mediante un análisis proximal con 5,71\% de proteína cruda y 1,06 Mcal/Kg MS de energía neta de ganacia de peso. No se encontraron diferencias significativas $(\mathrm{P}>0,05)$ en cada factor y en la interacción de estos, el grupo de animales menores de 25 meses de edad, tuvo un mejor peso promedio al final de la investigación con $308,48 \mathrm{Kg}( \pm 2,24)$, a la vez que los animales que fueron alimentados con una dieta que incluyó $20 \%$ de $\mathrm{CP}$ tuvieron un peso medio de $310,18 \mathrm{Kg}( \pm 2,10)$. Se concluye que la inclusión de compost con $20 \%$ CP en la alimentación de vaquillas menores de 25 meses no desfavorece la ganancia de peso y conversión alimenticia.

Palabras clave: Cerdaza, cascarilla de arroz, suplementación, rumiantes.

\begin{abstract}
This study evaluated the inclusion of deep bedding compost (DB) of rice husks and swine manure in the feeding of dual-purpose cross heifers. For this purpose, 18 heifers fed with DB processed in 12 months were used, obtained from the Teaching, Research and Outreach Unit of the Porcine Herd of the Escuela Superior Politécnica Agropecuaria de Manabí "Manuel Félix López". We applied a CRD with bifactorial arrangement [first factor: age (less than 25 months old and more than 25 months old and pregnant females at different stages of pregnancy); second factor: compost levels of DB $(0 \%, 10 \%$ and $20 \%)]$, which was considered as covariable to the initial weight. The nutritional composition of DB compost was determined through a proximal analysis with $5.71 \%$ of raw protein and $1.06 \mathrm{Mcal} / \mathrm{Kg} \mathrm{MS}$ of net energy of weight gain. No significant differences were found $(\mathrm{P}>0.05)$ among treatments and their interactions. The group of animals under 25 months of age had a better average weight at the end of the research with $308.48 \mathrm{~kg}( \pm 2.24)$, while animals that were fed a diet that included $20 \%$ of DB had an average weight of $310.18 \mathrm{~kg}( \pm 2.10)$. It is concluded that the inclusion of compost with $20 \%$ DB in the feeding of heifers under 25 months old does not affect weight gain and feed conversion.
\end{abstract}

Keywords: Swine manure, rice husks, supplementation, ruminants. 


\section{INTRODUCCIÓN}

La alimentación animal en el medio se ve limitado en algunas épocas por la escasez de pasturas o de materias primas para la elaboración del alimento balanceado o concentrado, por lo que se elevan los costos de producción y/o no se llegan a cumplir con los requerimientos nutricionales de las especies zootécnicas, donde afecta su desarrollo, producción de carne, leche y levante de las crías. Paralelamente a esto, la producción porcina genera excretas, que, además de servir como abono para fertilizar los suelos, sirven también para la alimentación animal; algunas investigaciones, indican que estas poseen algunos nutrientes de alto valor biológico que son utilizados por los rumiantes (Castrillón et al., 2004; Parra et al., 2007; Heredia, 2012).

A nivel mundial, el interés que ha tomado la crianza de especies bóvidas de interés zootécnico, ha encaminado a desarrollar alternativas de alimentación para los animales; sin embargo, el campo nutricional se desarrolla concomitantemente con la innovación informática y permanentemente alcanza nuevos niveles técnicos y científicos para la formulación de raciones, donde resulta más eficientes los sistemas de producción, donde se obtiene mayor cantidad de leche y carne de calidad y menores costos de la misma (FAO, 2018).

Ninabanda (2012) reporta que para aprovechar los contenidos nutricionales que se encuentran en la porquinaza, sería posible utilizar dicha materia orgánica como alternativa la conversión a proteína unicelular, luego de un proceso de descomposición natural, el cual, al eliminar los olores es posible utilizarlo en la nutrición de rumiantes. La porquinaza está compuesta por heces fecales y orina en unión al material que se emplea como cama (cascarilla de arroz), restos de alimento (balanceado), otras partículas y una cantidad de agua resultante de las pérdidas de los bebederos (Castrillón et al., 2004).

Según Fontemont et al. (1983) citados por Campabadal, (1994) describen que la porquinaza también es una fuente reconocida de proteína y minerales; de la misma forma Campabadal (1994) describe que en la composición de la porquinaza, el nutriente que más varía es la proteína, donde se han reportado pérdidas por volatilización de nitrógeno $(\mathrm{N})$, valores que fluctúan desde $11,62 \%$ hasta $32,5 \%$.; Lawrence (1971) y Sistani et al. (2001) citados por Jacobs et al. (2011) reportaron que disminuye la concentración de $\mathrm{N}$ en las heces cuando son secadas y cuando aumenta la temperatura. La mayor parte del fosforo (P) en las heces de los cerdos alimentados con granos de cereales, está presente en las excretas en forma de fosfato y solo pequeñas cantidades de fitato, a excepción de la dieta con maíz, en la que encontraron que el $45 \%$ del P total de las excretas estaba en forma de fitato (Leytem y Thacker, 2010).
Croker y Robinson (2002), argumentan que la genética influye en la cantidad de nutrientes excretados, las líneas maternas excretan en cantidades más bajas de todos los nutrientes (excepto amoniaco), que los cerdos F1 y de línea paterna, relacionado con un menor crecimiento de los cerdos del cruce Landrace x Large White. En la raza Pietrain, el efecto del genotipo halotano, codifica para un receptor del músculo esquelético, provocando en animales homocigotos recesivos y heterocigotos "mutante" una gran frecuencia de carnes pálidas, blandas y exudativas de muy baja calidad en la industria cárnica, adicional a esto, este gen tiene influencia sobre la excreción de $\mathrm{N}$ y $\mathrm{P}$, demostrando que los puros homocigotos recesivos y los heterocigotos para este gen, excretan menor concentración de $\mathrm{N}$ y $\mathrm{P}$ en comparación con los puros homocigotos dominantes (Saintilan et al., 2013).

Padilla et al. (2000) advierten que existen ciertos riesgos por el empleo de las excretas de aves y cerdos; por cuanto constituye un peligro sanitario para algunas especies animales y para el mismo hombre, ya que es natural que las excretas contengan elevadas cantidades de bacterias y hongos, por lo tanto, se deben tomar precauciones para evitar riesgos sanitarios; sin embargo, esta elevada presencia de microorganismos en las excretas no se considera como un peligro para la salud de los rumiantes que la consumen; ya que las condiciones de la fermentación que prevalecen en el rumen resultan ser adversas para la supervivencia de los microorganismos.

La FAO (2018), manifiesta que en América al igual que en todo el mundo, existe la necesidad de desarrollar tecnologías que permitan utilizar los recursos del medio, principalmente las excretas de los cerdos en la cama profunda, la misma que al ser manejada correctamente se aprovecha su valor nutricional en favor de la alimentación de rumiantes y por ende cumplir con los requerimientos nutricionales de los mismos a fin incrementar la producción y que los costos de producción no afecten al precio del producto terminado. Para la presente investigación se planteó como hipótesis nula que el promedio de las variables es igual entre grupos etarios y entre cada nivel de inclusión de compost de cama profunda de cascarilla de arroz y porquinaza (CP) y como objetivo evaluar el efecto de la inclusión de compost de cama profunda utilizada en la crianza de cerdos sobre la ganancia de peso de vaquillas mestizas de doble propósito.

\section{MATERIALES Y MÉTODOS}

\section{Localización y duración del experimento}

El desarrollo de esta investigación se realizó entre enero y marzo del 2019 con una duración de 10 semanas en la Unidad de Docencia, Investigación y Vinculación (UDIV) Hato Bovino de la carrera de Medicina Veterinaria perteneciente a la Escuela Superior Politécnica Agropecuaria de Manabí “Manuel Félix López” (ESPAM- 
MFL), ubicada en el sitio El Limón cantón Bolívar, provincia de Manabí, en las coordenadas $0^{\circ} 49^{\prime} 23$ " de latitud Sur y a $80^{\circ} 11^{\prime} 01^{\prime \prime}$ de longitud Oeste, a una altura de $15 \mathrm{msnm}$. En el transcurso de la investigación las variables climáticas promediaron en $26^{\circ} \mathrm{C}$, con humedad relativa de $81,40 \%$ y una precipitación media anual de $782,6 \mathrm{~mm}$.

\section{Unidades experimentales}

Se utilizaron 18 vaquillas mestizas de doble propósito de diferente composición racial con base Gyr y Pardo Suizo, distribuidas en grupos en función de la edad en grupo 1 menores a 25 meses de edad con promedio de peso de $248,89 \mathrm{Kg}( \pm 38,17)$ y grupo 2 mayores a 25 meses de edad y preñadas de diferente tiempo de gestación con media de peso de $318,11 \mathrm{Kg}( \pm 28,67)$, a las que se alimentó con raciones de balanceado con inclusiones de 0,10 y $20 \%$ de compost de cama profunda porcina (CP) en tres repeticiones. Los animales fueron alimentados una vez al día en horas de la tarde en corrales individuales de $3 \mathrm{~m}^{2}$, el resto del día consumieron pasto saboya (Panicum máximum).

\section{Procedimiento experimental}

Tres días antes de iniciar el ensayo, se procedió con la limpieza y desinfección de los corrales con agua y detergente y posteriormente se roció con una bomba de mochila con amonio cuaternario (CID-20 ®Carval), aplicando una dilución de 2,5ml/l de agua. Se alimentó a los animales a partir de las $14 \mathrm{H} 00$ con una ración de $1,0 \mathrm{Kg} / \mathrm{d} /$ animal con alimento balanceado de adaptación (Sin CP) durante 15 días y posteriormente se suministró $1,5 \mathrm{Kg} / \mathrm{d} /$ animal de alimento balanceado durante ocho semanas según el tratamiento correspondiente, incluyendo en la dieta CP de la UDIV Hato Porcino de la ESPAMMFL, el mismo que tuvo un proceso natural de compostaje de alrededor de un año.

Previo a la preparación del alimento, se determinó el valor nutritivo de CP con muestras enviadas al laboratorio de servicio de análisis e investigación en alimentos del Instituto Nacional de Investigaciones Agropecuarias (INIAP), acantonado en la Estación Experimental Santa Catalina en la provincia de Pichincha, en la que se realizó un análisis proximal de humedad (Método MO-LSAIA01.01), cenizas (Método MO-LSAIA-01.02), extracto etéreo (Método MO-LSAIA-01.03), proteína cruda (Método MO-LSAIA-01.04), fibra cruda (Método MOLSAIA-01.05) y extracto libre de nitrógeno (Método MOLSAIA-01.06), considerando en todos el método de referencia de la Universidad de Florida 1970. La energía neta de ganancia de peso $(\mathrm{ENg})$ fue calculada a través de ecuaciones a partir de la cantidad de fibra cruda encontrada en el análisis proximal de $\mathrm{CP}$, previo a este cálculo se estimó nutrientes digeribles totales (NDT), energía digerible (ED) y energía metabolizable (EM).

$$
\text { NDT }=710-0,43 \text { FC (Donker, 1989) (1) }
$$

Donde:

NDT $=$ Nutrientes digeribles totales $(\mathrm{g} / \mathrm{Kg}$ de materia seca)

$\mathrm{FC}=$ Fibra cruda $(\mathrm{g} / \mathrm{Kg}$ de materia seca)

MS = Materia seca

$$
1 \mathrm{Kg} \text { NDT }=4,409 * \mathrm{ED} \text { (Weiss, 1993) (2) }
$$

Donde:

NDT $=$ Nutrientes digeribles totales $(\mathrm{Kg})$

$\mathrm{ED}=$ Energía digerible (Mcal/Kg de materia seca)

$$
\mathrm{EM}=-0,45+1,01 * \mathrm{ED}(\text { Weiss, 1993) (3) }
$$

Donde:

$\mathrm{EM}=$ Energía metabolizable (Mcal/Kg de materia seca)

$\mathrm{ED}=$ Energía digerible (Mcal/Kg de materia seca)

$$
\begin{gathered}
\mathrm{ENg}=1,42 * \mathrm{EM}-1,74 * \mathrm{EM}^{2}+0,0122 \mathrm{EM}^{3}-1.65 \\
(\mathrm{NRC}, 2001)(4)
\end{gathered}
$$

Donde:

$\mathrm{ENg}$ = Energía neta de ganancia de peso $(\mathrm{Mcal} / \mathrm{Kg}$ de materia seca)

$\mathrm{EM}=$ Energía metabolizable (Mcal/Kg de materia seca)

El alimento se elaboró de acuerdo al peso y a los requerimientos nutricionales recomendados por la NRC (2000), el mismo que se preparó en la planta de balanceados de los talleres agroindustriales de la UDIV de la ESPAM-MFL, en el que se consideró cada uno de los niveles de inclusión de $\mathrm{CP}$ en la dieta experimental correspondiente como se observa en el cuadro 1.

Cuadro 1. Composición de materias primas (\%) de las dietas experimentales.

\begin{tabular}{lccc}
\hline \multirow{2}{*}{\multicolumn{1}{c}{ Ingredientes }} & \multicolumn{3}{c}{ Nivel de inclusión } \\
\cline { 2 - 4 } & $\mathbf{0 \%}$ & $\mathbf{1 0 \%}$ & $\mathbf{2 0 \%}$ \\
\hline Maíz nacional & 15,00 & 12,50 & 9,57 \\
Afrecho de trigo & 30,00 & 23,98 & 24,42 \\
Polvillo de arroz & 19,86 & 30,00 & 30,00 \\
Palmiste & 10,00 & 10,00 & 10,00 \\
Melaza de caña & 22,50 & 11,84 & 4,97 \\
Sal mineral & 0,62 & 0,00 & 0,00 \\
Carbonato de calcio & 2,00 & 1,69 & 1,04 \\
Compost de cama profunda & 0,00 & 10,00 & 20,00 \\
\hline Total & 100,00 & 100,00 & 100,00 \\
\hline
\end{tabular}

Dentro de cada grupo etario los animales fueron distribuidos aleatoriamente en cada unidad experimental 
con su respectivo nivel de inclusión de CP; todos los tratamientos fueron sometidos al mismo manejo.

El alimento se distribuyó individualmente a cada animal en comederos. Los animales fueron pesados semanalmente en una báscula electrónica (Tru-Test MP 600 ® Eeighing \& Eid) con capacidad hasta $2000 \mathrm{Kg}$ ubicado dentro de un trinquete de sujeción de $2 \mathrm{~m}$ de longitud por 0,80 $\mathrm{m}$ de ancho en la UDIV Hato Bovino de la ESPAM-MFL.

\section{Diseño experimental}

Se aplicó un diseño completamente aleatorizado con arreglo bifactorial considerando como primer factor dos grupos etarios y como segundo factor tres niveles de inclusión de CP; se incluyó como covariable el peso inicial para las variables ganancia de peso y conversión alimenticia, valores que fueron procesados por medio del paquete estadístico InfoStat (2018).

\section{RESULTADOS Y DISCUSIÓN}

\section{Caracterización del contenido nutricional del CP}

Los resultados obtenidos de la composición de CP utilizada en la alimentación de vaquillas mestizas de doble propósito luego de un proceso de compostaje por un lapso de 12 meses aproximadamente, tomando en considerando que contenía un alto porcentaje de cascarilla de arroz, los valores nutricionales resultaron bajos (cuadro 2), en comparación a otras investigaciones que utilizaron porquinaza pura. La composición nutricional de la porquinaza es afectada principalmente por las variaciones en la formulación de las dietas utilizadas, el método de procesamiento y manejo del producto, la etapa productiva, el ambiente, el manejo de los cerdos y el método de recolección (Castrillón et al., 2004; Campabadal, 1995 citado por Ávalos, 2014).

Cuadro 2. Análisis proximal de la cama profunda porcina

\begin{tabular}{lccc}
\hline \multicolumn{1}{c}{ Nutrientes } & CP* & $\begin{array}{c}\text { Romero } \\
(\mathbf{2 0 1 6})\end{array}$ & $\begin{array}{c}\text { Ávalos } \\
(\mathbf{2 0 1 4})\end{array}$ \\
\hline Humedad (\%) & 9,62 & 7,99 & 18,68 \\
Cenizas (\%) & 66,84 & 18,25 & 7,66 \\
Extracto Etéreo (\%) & 0,34 & 6,95 & 2,98 \\
Proteína cruda (\%) & 5,71 & 23,26 & 11,68 \\
Fibra cruda (\%) & 7,07 & 13,72 & 11,58 \\
$\begin{array}{l}\text { Extracto libre de } \\
\text { nitrógeno (\%) }\end{array}$ & 20,05 & 29,83 & 56,59 \\
$\begin{array}{l}\text { Energía neta ganancia } \\
\text { de peso (Mcal/kg)** }\end{array}$ & 1,06 & --- & --- \\
\hline
\end{tabular}

*Laboratorio de Servicio de Análisis e Investigación en Alimentos del Instituto Nacional de Investigaciones Agropecuarias (2018); ** Valor calculado.
La porquinaza en etapas de inicio, presenta un elevado contenido de proteína cruda (PC), extracto etéreo (EE), carbohidratos no estructurales (CNE) y energía que la porquinaza de los animales reproductores (gestantes y lactantes) debido al mayor uso de los nutrimentos de la dieta por parte de los cerdos jóvenes (Castrillón et al., 2004). Estos resultados podrían estar relacionados con el nivel de proteínas que requieren los animales jóvenes, y podría atribuirse a que muchos nutrientes no son asimilados en su totalidad y son excretados mediante heces y orina y adicional se considera los desperdicios del alimento en la $\mathrm{CP}$.

\section{Efecto de la inclusión de CP sobre el peso.}

En el cuadro 3, se observa que no se evidenciaron diferencias significativas $(p>0,05)$ entre los grupos etarios durante las ocho semanas que duró la investigación; sin embargo, se reportan pesos finales similares de $309,41 \mathrm{Kg}$ $( \pm 2,24)$ y $308,48( \pm 2,24)$ para las vaquillas menores a 25 meses de edad y mayores a 25 meses de edad en su orden.

Cuadro 3. Peso semanal en función del grupo etario $(\mathrm{Kg})$

\begin{tabular}{cccc}
\hline \multirow{2}{*}{ Semana } & \multicolumn{2}{c}{ Edad } & p-valor \\
\cline { 2 - 3 } & $<25$ meses & $>25$ meses & \\
\hline 1 & $283,02( \pm 2,48)$ & $282,21( \pm 2,48)$ & 0,8492 \\
2 & $286,97( \pm 2,40)$ & $287,53( \pm 2,40)$ & 0,8939 \\
3 & $297,44( \pm 2,88)$ & $291,56( \pm 2,88)$ & 0,2502 \\
4 & $300,21( \pm 2,78)$ & $297,18( \pm 2,78)$ & 0,5295 \\
5 & $302,79( \pm 2,92)$ & $302,65( \pm 2,92)$ & 0,9786 \\
6 & $305,70( \pm 3,02)$ & $304,58( \pm 3,02)$ & 0,8296 \\
7 & $307,66( \pm 2,55)$ & $307,34( \pm 2,55)$ & 0,9401 \\
8 & $309,41( \pm 2,24)$ & $308,48( \pm 2,24)$ & 0,8089 \\
\hline
\end{tabular}

p-valor $=$ Valor de probabilidad

Morales et al. (2002) citados por Ramírez (2015), reportan que los pesos en vaquillas presentaron comportamiento lineal (sin variabilidad de datos) para cada uno de los tratamientos aplicado (solo pasto, pollinaza, porquinaza y balanceado comercial), lo cual concuerda con los resultados de esta investigación por un efecto normal del desarrollo de los animales jóvenes y la ganancia de peso de los animales adultos. Romero (2016) indica que la porquinaza es considerada una reconocida fuente de proteína y minerales que puede ser aprovechada todo el año lo cual explica el efecto de la proteína en una mejor ganancia de peso en animales jóvenes, considerando que al momento de iniciar la investigación los pesos fueron variados y la condición corporal promedio alrededor de 2 a 2,5 en la escala de 1 a 5 para bovinos de leche propuesta por Yukun et al. (2019). 
El INIA (2006) revela que mientras más avanzada la edad, el aumento de peso en el animal adulto estará representado por una mayor proporción de grasa en la composición química de la ganancia de peso y en jóvenes estará dado por una mayor proporción de proteína (músculo) en desmedro de la grasa; el depósito de grasa en el cuerpo, le significa al animal un mayor costo energético que si deposita proteína, por ello, los animales adultos deben aumentar su consumo de alimentos para compensar su mayor ineficiencia en el uso de energía.

Cerdas (2013) indica que el mantenimiento y crecimiento de los bovinos requiere proteína verdadera absorbida en el intestino y energía en los tejidos en proporciones adecuadas, además de la edad animal ya que si son animales jóvenes tendrán una mayor ganancia de peso que los animales longevos y por otro lado la genética, ya que indistintamente los bovinos destinados a carne obtendrán más peso que los destinados a la producción de leche.

En cuanto al nivel de utilización, no se encontró diferencia significativa $(p>0,05)$ entre los diferentes niveles de utilización de CP; sin embargo, se observó que las vaquillas que consumieron balanceado con $20 \%$ de CP obtuvieron mayor peso promedio durante el experimento con una media final de $310,18 \mathrm{Kg}( \pm 2,10)$ (Cuadro 4).

Cuadro 4. Peso semanal en función del nivel de inclusión de $\mathrm{CP}$ en la dieta $(\mathrm{Kg})$

\begin{tabular}{|c|c|c|c|c|}
\hline \multirow{2}{*}{ Semana } & \multicolumn{3}{|c|}{ Nivel de CP } & \multirow{2}{*}{ p-valor } \\
\hline & $\mathbf{0}$ & 10 & 20 & \\
\hline 1 & $282,46( \pm 2,33)$ & $281,44( \pm 2,32)$ & $283,93( \pm 2,32)$ & 0,7547 \\
\hline 2 & $286,26( \pm 2,25)$ & $286,73( \pm 2,25)$ & $288,76( \pm 2,25)$ & 0,7145 \\
\hline 3 & $292,04( \pm 2,70)$ & $294,23( \pm 2,70)$ & $297,23( \pm 2,70)$ & 0,4238 \\
\hline 4 & $296,85( \pm 2,61)$ & $298,19( \pm 2,61)$ & $301,04( \pm 2,60)$ & 0,5305 \\
\hline 5 & $301,15( \pm 2,75)$ & $302,14( \pm 2,74)$ & $304,88( \pm 2,74)$ & 0,6230 \\
\hline 6 & $303,74( \pm 2,84)$ & $304,99( \pm 2,83)$ & $306,68( \pm 2,83)$ & 0,7685 \\
\hline 7 & $306,30( \pm 2,39)$ & $306,79( \pm 2,39)$ & $309,41( \pm 2,39)$ & 0,6280 \\
\hline 8 & $308,50( \pm 2,11)$ & $308,16( \pm 2,10)$ & $310,18( \pm 2,10)$ & 0,7738 \\
\hline
\end{tabular}

p-valor $=$ Valor de probabilidad

Parra et al. (2007), en un experimento de inclusión de tres niveles de porquinaza $(0 \%, 25 \%$ y $50 \%)$ en corderos Hamshire en la etapa de acabado, no encontró diferencia significativa ( $>>0,05)$, no obstante, el tratamiento con la inclusión de $25 \%$ de porquiza en la dieta, fue en el que obtuvo la mayor ganancia diaria de peso con 203,5 g/día.

Campabadal (1994) citado por Ramírez (2015), manifiesta que al comparar porquinaza seca y fresca, concluye que en la alimentación del ganado de carne con un $60 \%$ de porquinaza en la dieta combinada con otros subproductos industriales pueden oscilar las ganancias de peso entre 0,5 y $0,9 \mathrm{Kg} /$ día, según la genética del animal, lo que concuerda con el efecto observado en este experimento por lo que se asume que el valor biológico en la proteína de la porquinaza permitió una mayor ganancia de peso en los animales < de 25 meses de edad que consumieron una mayor cantidad de $\mathrm{CP}$.
Según González et al. (2010) y García (2000) citado por Ramírez (2015), indican que al analizar en pruebas micotóxicas, la toxicidad de la porquinaza deshidratada resulta tres veces menos tóxica que la pollinaza, reduce la emisión de olores y la presencia de Salmonella typhimurium hasta en $99,98 \%$. La cerdaza con frecuencia contiene altas concentraciones de $\mathrm{Cu}$ y $\mathrm{Zn}$ comparado con estiércoles de otras especies, debido a que el $\mathrm{Cu}$ se adiciona a las raciones, con el fin de aumentar la ganancia de peso y la conversión alimenticia; asimismo el Zn se utiliza para contrarrestar el potencial de toxicidad de $\mathrm{Cu}$ (García, 2000 citado por Ávalos, 2014).

En el cuadro 5, se observó que no existen diferencias significativas $(p>0,05)$ entre las interacciones para la variable peso; Sin embargo, los animales menores de 25 meses de edad suplementados con $20 \%$ de CP obtuvieron los mayores pesos promedio con $313,44 \mathrm{Kg}( \pm 3,13)$ al finalizar el experimento e incluso sobre los animales mayores a 25 meses de edad, lo que refleja la capacidad del desarrollo compensatorio aprovechado con la dieta. 
Cuadro 5. Peso semanal en función de la interacción de la edad y el nivel de inclusión en la dieta $(\mathrm{Kg})$

\begin{tabular}{|c|c|c|c|c|c|}
\hline \multirow{2}{*}{ Sem. } & \multirow{2}{*}{ Edad } & \multicolumn{3}{|c|}{ Nivel de CP } & \multirow{2}{*}{ p-valor } \\
\hline & & 0 & 10 & 20 & \\
\hline \multirow{2}{*}{1} & $<25$ & $282,89( \pm 4,12)$ & $279,22( \pm 3,49)$ & $286,95( \pm 3,45)$ & \multirow{2}{*}{0,3184} \\
\hline & $>25$ & $282,03( \pm 4,03)$ & $283,67( \pm 3,42)$ & $280,92( \pm 3,59)$ & \\
\hline \multirow{2}{*}{2} & $<25$ & $286,20( \pm 3,99)$ & $284,39( \pm 3,38)$ & $290,34( \pm 3,35)$ & \multirow{2}{*}{0,4908} \\
\hline & $>25$ & $286,33( \pm 3,90)$ & $289,07( \pm 3,31)$ & $287,19( \pm 3,48)$ & \\
\hline \multirow{2}{*}{3} & $<25$ & $297,80( \pm 4,79)$ & $294,79( \pm 4,06)$ & $299,73( \pm 4,01)$ & \multirow[b]{2}{*}{0,4723} \\
\hline & $>25$ & $286,28( \pm 4,69)$ & $293,66( \pm 3,98)$ & $294,74( \pm 4,18)$ & \\
\hline \multirow{2}{*}{4} & $<25$ & $300,67( \pm 4,63)$ & $296,58( \pm 3,92)$ & $303,39( \pm 3,88)$ & \multirow{2}{*}{0,3837} \\
\hline & $>25$ & $293,02( \pm 4,53)$ & $299,80( \pm 3,84)$ & $298,70( \pm 4,03)$ & \\
\hline \multirow{2}{*}{5} & $<25$ & $303,24( \pm 4,87)$ & $298,21( \pm 4,12)$ & $306,91( \pm 4,08)$ & \multirow{2}{*}{0,2657} \\
\hline & $>25$ & $299,05( \pm 4,76)$ & $306,06( \pm 4,04)$ & $302,85( \pm 4,24)$ & \\
\hline \multirow{2}{*}{6} & $<25$ & $304,96( \pm 5,03)$ & $301,73( \pm 4,27)$ & $310,41( \pm 4,22)$ & \multirow{2}{*}{0,2568} \\
\hline & $>25$ & $302,53( \pm 4,92)$ & $308,25( \pm 4,18)$ & $302,96( \pm 4,39)$ & \\
\hline \multirow{2}{*}{7} & $<25$ & $305,54( \pm 4,24)$ & $304,70( \pm 3,59)$ & $312,75( \pm 3,55)$ & \multirow{2}{*}{0,2864} \\
\hline & $>25$ & $307,07( \pm 4,15)$ & $308,87( \pm 3,52)$ & $306,06( \pm 3,70)$ & \\
\hline \multirow{2}{*}{8} & $<25$ & $308,03( \pm 3,73)$ & $306,77( \pm 3,16)$ & $313,44( \pm 3,13)$ & \multirow{2}{*}{0,2941} \\
\hline & $>25$ & $308,96( \pm 3,65)$ & $309,55( \pm 3,10)$ & $306,91( \pm 3,25)$ & \\
\hline
\end{tabular}

p-valor $=$ Valor de probabilidad

\section{Efecto de la inclusión de CP sobre la conversión alimenticia}

Al analizar la conversión alimenticia (CA) del balanceado sobre el efecto de la edad de los animales, no se encontró diferencia significativa $(p>0,05)$ entre los grupos etarios los mismos que se encontraron valores de $3,36( \pm 0,22)$ y $3,47( \pm 0,22)$ para los animales menores de 25 meses y mayores que estos últimos en su orden. De la misma forma al analizar el nivel inclusión de CP en la dieta, no se encontró diferencia significativa ( $p>0,05)$, se halló que al nivel de $20 \%$ de CP, la CA fue de $3,26( \pm 0,27)$ siendo la más baja, seguido de la dieta sin $\mathrm{CP}$ con $3,45( \pm 0,27)$ y con el nivel de $10 \%$ de CP se obtuvo un valor más alto de CA con 3,53 ( $\pm 0,27)$; en la interacción entre la edad y el nivel de $\mathrm{CP}$, no se detectó diferencia significativa $(\mathrm{p}>0,05)$ entre la interacción de estos factores, las vaquillas menores a 25 meses de edad y que consumieron $20 \%$ de CP, mostraron el menor promedio de CA con $2,84( \pm 0,39)$.

Sánchez (2015) describe que animales jóvenes tienen una mejor conversión alimenticia que los animales longevos, ya que los animales realimentados depositan tejidos de un contenido calórico menor (poca grasa y mucho músculo), por lo que se considera que esto sería un factor importante en la determinación de una eficiencia mayor durante ese período, ya que la proteína tiene un valor calórico menor que el de la grasa y se supone que la compensación es debida a crecimiento muscular. Heredia (2012) concluye que el suministro de porquinaza en terneros de levante influye en la conversión alimenticia, permitiendo utilizar el valor biológico de la proteína de la cerdaza.

\section{Análisis económico}

Se obtuvo un mayor ingreso en animales menores a 25 meses de edad con inclusión de compost del $20 \%$ los que por cada dólar invertido lograron un beneficio de 0,91 dólares americanos (USD), en tanto que al $0 \%$ de porquinaza alcanzaron 0,33 USD; al contrario, los animales que recibieron el $10 \%$ lograron la menor ganancia ( $\$ 0,15$ USD). En los animales mayores a 25 meses con inclusión de $10 \%$ de porquinaza obtuvo una ganancia de 0,45 USD; seguido el $20 \%$ con 0,30 USD y por último $0 \%$ con 0,09 USD por cada dólar invertido.

Morales et al. (2002) citados por Ramírez (2015), indican que tanto la pollinaza como la porquinaza, son insumos que abaratan los costos y eleva la ganancia de peso en vaquillas mestizas, esto se podría atribuir al factor biológico, que podría ser la razón por la cual ciertas vaquillas no presentaron un peso semanal con mucha variabilidad.

\section{CONCLUSIONES}

El efecto de la inclusión el compost proveniente de la cama profunda de hato porcino en la alimentación de vaquillas mestizas de doble propósito, no se encontraron diferencias significativas $(P>0,05)$ en cada factor y en la interacción de estos; se destacó la ganancia de peso e índice de conversión alimenticia con la inclusión del nivel correspondiente al $20 \%$ en animales menores a 25 meses de edad; también el nivel de inclusión del $20 \%$ de CP resultó ser la interacción que mayor beneficio económico rindió con 0,91 USD por dólar invertido. 
Al incluir niveles de suplementación de porquinaza en el la alimentación de vaquillas, no se observó rechazo del alimento por lo que un buen proceso de secado y compostaje permite que sea palatable al ser suministrado con otras materias primas especialmente melaza.

\section{LITERATURA CITADA}

Ávalos, A. 2014. Evaluación de tres formas para la fermentación de cerdaza. (En línea). Zamorano, GT. Consultado, 18 de oct. 2017. Formato PDF. Disponible: http://www.repositorio.usac.edu.gt/1574/

Campabadal, C. 1994. Utilización de la porquinaza en la alimentación del ganado de carne. Una alternativa para evitar la contaminación ambiental. San José, CR. Nutrición Animal Tropical. 3 (1): 73-96.

Castrillón, O. Jiménez, R. Bedoya, O. 2004. Porquinaza En La Alimentación Animal. CO. Revista Lasallista de Investigación. 1 (1): 72-76.

Cerdas, R. 2013. Formulación de raciones para carne y leche. Desarrollo de un módulo práctico para técnicos y estudiantes de ganadería de Guanacaste, Costa Rica. Guanacaste, CR. Revista de las Sedes Regionales. 14 (29): 128-153.

Crocker, A. Robinson, O. 2002. Ganetic and nutritional effects on swine excreta. Journal of Animal Science. 80 (11): $2809-2816$

Donker, J. 1989. Improve energy prediction equations for dairy cattle rations. J. Dairy Sci. 72: 2942-2948.

INIA (Instituto Nacional de Investigaciones Agropecuarias). 2006, Manual de producción de leche para pequeños y medianos productores, Boletín INIA $\mathrm{N}^{\circ}$ 148, http://biblioteca.inia.cl/medios/biblioteca/ boletines/NR33823.pdf

FAO (Organización de las Naciones Unidas para la Alimentación y la Agricultura). 2018. Producción Animal. Papel la FAO en la producción animal. (En línea). Consultado, 18 de oct. 2017. Disponible: http://www.fao.org/animal-production/es/

Heredia, M. 2012. Utilización de porquinaza en dietas de levante para terneros pos destete. (En línea). Zamorano, HN. Consultado, 18 de oct. 2017. Formato PDF. Disponible: https://bdigital.zamorano.edu/ bitstream/11036/1256/1/T3298.pdf

Jacobs, B. Patience, J. Dozier, W. Stalder, Kerr, B. 2011. Effects of drying methods on nitrogen and energy concentrations in pig feces and urine, and poultry excreta. Journal of Animal Science. 89 (8): $2624-$ 2630.
Leytem, A., and Thacker, P. 2010. Phosphorus utilization and characterization of excreta from swine fed diets containing a variety of cereal grains balanced for total phosphorus. Journal of Animal Science. 88: 18601867.

Ninabanda, J. 2012. Alternativas de manejo de las excretas porcinas. (En línea). Riobamba, EC. Consultado, 18 de oct. 2017. Formato PDF. Disponible: http:// http://dspace.espoch.edu.ec/handle/123456789/2109

NRC (National Research Council). 2001. Nutrients requirements of dairy cattle. (Séptima edición). National Academy Press, USDA, Washington, DC.

Padilla, E., Castellanos, A., Cantón, J., Moguel, Y. 2000. Impacto del uso de niveles elevados de excretas animales en la alimentación de ovinos. Merida, MX. Livestock Research for Rural Development. 12 (1).

Parra, C. Martínez, J. Rincón, J. 2007. Utilización de porquinaza en dos niveles de inclusión, como suplemento alimenticio en la etapa de finalización en el período de engorde en ovinos. (En línea). Consultado, 18 de feb. 2019. Formato PDF. Disponible: https:// https://repository.unad.edu.co/ handle/10596/1451

Ramírez, M. 2015. Evaluación económica del engorde de toretes alimentados con porquinaza; pollinaza y concentrado comercial. (En línea). Cuenca, EC. Consultado, 18 de feb. 2019. Formato PDF. Disponible: https://publicaciones.ucuenca.edu.ec/ojs/ index.php/maskana/article/view/677

Romero, T. 2016. Evaluación del incremento de peso en bovinos mestizos con pollinaza y porquinaza como suplementación alimenticia en el cantón Marcabeli. (En línea). Machala, EC. Consultado, 18 de feb. 2019. Formato PDF. Disponible: http:// http://repositorio. utmachala.edu.ec/handle/48000/7693

Saintilan, R., Mérour, I., Brossard, L., Tribout, T., Dourmad, J., Sellier, P., Bidanel, J., Van Milgen, J., Gilbert, H. 2013. Genetics of residual feed intake in growing pigs: Relationships with production traits, and nitrogen and phosphorus excretion traits. Journal of Animal Science. 91: 2542-2554.

Weiss, W. 1993. Predicting energy values of feeds. J. Dairy Sci. 76: 1802-1811.

Yukun, S., Pengju, H., Yujie, W., Ziqi, C., Yang, J., Baisheng, D., Runze, L., Yonggen, Z. 2019. Automatic monitoring system for individual dairy cows based on a deep learning frameworks that provides identification via body parts and estimation of body condition score. J. Dairy Sci. 102 (11): 10140-10151. 